\title{
Escalation of Efficacy and Prevention of Chemoresistance in Various Cancer Therapies by the Utilization of Targeting the Crosstalk Amongst MAPK/ERK Along with Hippo/MST Signaling - A Comprehensive Review
}

\author{
Kulvinder Kochar Kaur ${ }^{1 *}$, Gautam Allahbadia ${ }^{2}$ and Mandeep Singh ${ }^{3}$ \\ ${ }^{1}$ Scientific Director, Dr Kulvinder Kaur Centre for Human Reproduction, Jalandhar, \\ Punjab, India \\ ${ }^{2}$ Scientific Director, Rotunda-A Centre for Human Reproduction, Mumbai, India \\ ${ }^{3}$ Consultant Neurologist, Swami Satyan and Hospital, Jalandhar, Punjab, India \\ *Corresponding Author: Kulvinder Kochar Kaur, Scientific Director, Dr Kulvinder \\ Kaur Centre for Human Reproduction, Jalandhar, Punjab, India.
}

Received: August 19, 2021

Published: August 31, 2021

(C) All rights are reserved by Kulvinder

Kochar Kaur., et al.

\begin{abstract}
Earlier having reviewed the treatment of ovarian cancer, endometrial carcinoma, gestational trophoblastic disease ( GTD), along with colorectal cancer, breast cancer etc, here we decided to comprehensively evaluate the crosstalk of various signaling pathway implicated in cancer therapy, in particular how the crosstalk amongst mitogen activated protein kinase (MAPK)/extracellular signal -regulated kinase (ERK1/2) signaling pathway with the Hippo/MST signaling pathway knowledge might impact on the generation of newer therapies by getting insight into the harms of physiology of phosphatidyl inositide 3-kinase (PI3K)/activator of protein kinase B (AKT/mammalian target of rapamycin (mTOR) pathway and combining therapies targeting Hippo/MST signaling pathway might avoid in the generation of chemoresistance. In particular the ERK represents a part of the MAPK signaling pathway that is amarkedly conserved pathway of signal transduction. This MAPK gets constituted by three separate kinases, like RAF/MEK as well as ERK. Impairment of the MAPK signaling takes place in a lot of pathophysiological conditions that are inclusive of neurodegenerative diseases, (NGD), Metabolic Syndrome (MetS) in addition to lot of cancers. Targeted hampering of the unique kinases belonging to the MAPK signaling pathway with the utilization of certain synthetic agents might work out to be of significant advantage for the treatment of cancer. Interaction of the MAPK signaling pathway with other proteins in addition to signaling pathways possess a key influence on the Clinical results of targeted treatments, besides possessing a significant part at the time of generation of drug resistance In cancers. Here we have tried to detail the interaction of the MAPK signaling pathway with rest of the signaling pathways specifically its crosstalk with the Hippo/MST signaling pathway, besides detailing how it may prove advantageous on utilization of targeting of these particular pathways In combination in the generation of anticancer treatments that turn out to possess greater efficacy.
\end{abstract}

Keywords: MAPK; ERK; Hippo; MST; PI3K; YAP; Cancer; Caspases; Natural Agents; Apoptosis

\section{Introduction}

The MAPK/ERK path is considered a marked conserved pathway regarding signal transduction made of 3 separate kinases, known as RAF, MEK as well as ERK [1]. To begin with MAPK/ERK path was detailed initially In the form of a transducer of extracellu- lar signals via epidermal growth factor receptor (EGFR) to extracellular signal -regulated kinase (ERK1/2) ERK1/2 kinase phosphorylates various protein substrates that take part In the control of basal cellular functions that are survival, differentiation, as well as metabolism. Dysregulation of this MAPK/ERK signaling path takes 
Escalation of Efficacy and Prevention of Chemoresistance in Various Cancer Therapies by the Utilization of Targeting the Crosstalk Amongst MAPK/ERK Along with Hippo/MST Signaling - A Comprehensive Review

place in various kinds of malignancies making parts of the MAPK/ ERK signaling path as a potential area for targeting regarding treatment of various cancers [1,2] see figure 1 .

Generation of resistance within cells in the cancers is one of the main restrictions at the time of monotherapy with the utilization of single MAPK/ERK inhibitor. Cause of resistance generation is secondary to MAPK/ERK crosstalk with separate signaling pathways like phosphatidyl inositide 3-kinase (PI3K)/activator of protein kinase B (AKT), mammalian target of rapamycin (mTOR) signaling pathway [3]. Conversely various publications detail a key part regarding activation of MAPK/ERK signaling path at the time of cell death stimulation in broad spectrum of cancer cells pointing tumor repressor activity of the MAPK/ERK signaling path. Parts of the Hippo/MST signaling were shown to be targets of MEK as well as ERK kinase giving proof regarding tumor repressor activity of the MAPK/ERK signaling in malignant cells.

Here we try to emphasize on the effect of the MAPK/ERK crosstalk with rest of the signaling pathways in addition to MAPK/ERK protein crosstalk in the control of single cellular events in cancer cells. Basically concentration on crosstalk among MAPK/ERK, Hippo/MST along with PI3K/AKT/mTOR signaling pathways In differentiation of cancer cells in addition to detail crosstalk among these signaling pathways considering the present cancer treatments. Emphasis on sameness among MAPK/ERK, along with Hippo/MST signaling pathways In control of proliferation along with stimulation of cell death in cancer cells. Then combination targeting of MAPK/ ERK, Hippo/MST, along with PI3K/AKT/mTOR signaling pathways for bettering the results of recent cancer treatment is required.

\section{MAPK/ERK signaling pathways-canonical}

The canonical MAPK/ERK pathway is constituted of three kinds of MAPK Kinase Kinase (MAPKKK) A-RAF, B-RA and RAF-1 or CRAF kinases. BRAF is the gene most commonly mutated at this level in human cancer. One level downstream are the): (MAPK Kinase (MAPKKs), which are composed of MEK1 and MEK2. Finally, further below are ERK1 and ERK2, which are the final effectors of the MAPK pathway (Figure 1). MAPK1/ERK2 in addition to MAPK3/ ERK1 are 2 serine/threonine kinases that have been well understood to be implicated in the manipulation of the MAPK Signal transduction pathway. ERK 1along with ERK2 kinase work as the final signal node of the MAPK/ERK signaling pathways, besides participating in the control of cellular events like proliferation, metabolism in addition to survival. ERK1/2 kinase phosphorylates a wide spectrum of protein substrates implicated. In transcriptional control, cytoskeleton organization, protein phosphorylation, protein translation in addition to cell death induction. The Canonical (conforming to a general rule) MAPK Signal Transduction pathway is usually show as a positive Controller of cellular proliferation, growth along with survival. Selectively inhibiting individual kinases of MAPK Signal Transduction pathway, utilized targeted inhibitors is a good way of treatment of cancer efficiently yet resistance form is a robust restriction [1].

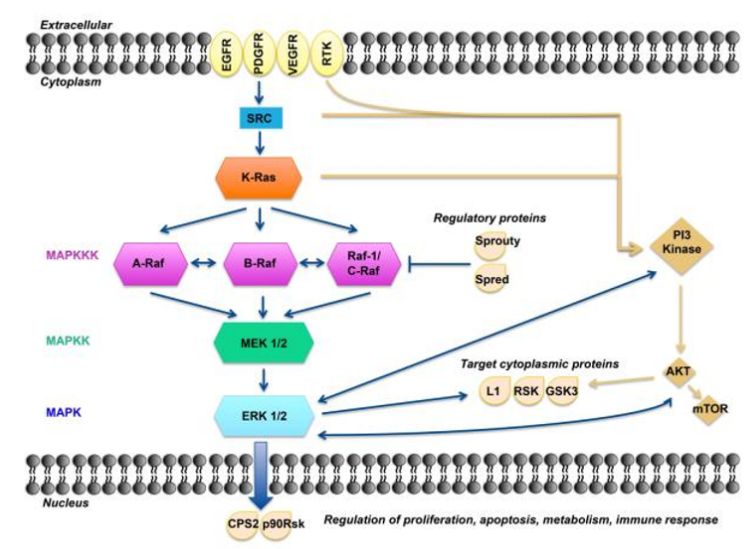

Figure 1: Courtesy ref no-3 - A model of the MAPK/ERK pathway. After membrane receptor activation, adaptor proteins recruit RAS proteins to activate steps concluding with ERK activation. Successive steps of phosphorylation amplify the signal, $\mathrm{Raf} \rightarrow \mathrm{MEK} \rightarrow \mathrm{ERK}$, until ERK activates its cytoplasmic and/or nuclear targets. Regulatory phosphatases, Sprouty and Spred, modulate the intensity of the signal. The PI3K-AKT pathway interacts with the MAPK/ERK node under normal conditions and in the cancer cell. Target cytoplasmic proteins include RSK, ribosomal S6 kinases; GSK3, glycogen synthase kinase 3; L1, adhesion molecule L1. Additional proteins in nucleus include CPS2, p90Rsk.

Known ERK kinase crosstalk-Identity as well as function

ERK kinases possess sharing of lot of protein interactors. Bio GRID databases consists of 270 unique interactors of ERK 2 ki-

Citation: Kulvinder Kochar Kaur., et al. "Escalation of Efficacy and Prevention of Chemoresistance in Various Cancer Therapies by the Utilization of Targeting the Crosstalk Amongst MAPK/ERK Along with Hippo/MST Signaling - A Comprehensive Review". Acta Scientific Cancer Biology 5.9 (2021):

37-47. 
nases [3]. Nevertheless, the correlation of 'lot of interactors with ERK 2 kinases came via outcomes got through utilization of high throughput techniques (HTT) or hit only in a single proof. Filtration of interactors depending on less than 3 proofs in addition to interactors tagged as high throughput mined approximately 50 protein interactors which are proven targets of the ERK kinases (Figure 2) [4]. These interactors implicate 1) controllers of transcription (ATF2, STAT5, SP1, JUN, HIF1A, MYC, ELK1, FOXO3, SMAD1, HDAC along with EP300) 2) Protein kinases (MAPK14, RAF1, NEK2, MAP2, MKNK2, MAP2K7, MAP2K2, MAPK3, MAP2K1, MKNK1, MAP3K1, RPS6KA1, GSK3B, RPS6KA3, RPS6KA2, RPSKA4. 3) Protein phosphates (PTPN5, PTPRC, PTPN7, DUSP1, DUSP6, DUSP9) 4) Regulators of Apoptosis (DAPK1, TFRS1A, TP53, PPARG) 5) GTPase activity correlated Proteins (KRAS, TSC2, RPTOR). Control of these protein crosstalk by ERK kinases give varied outputs with regards to cellular functions.

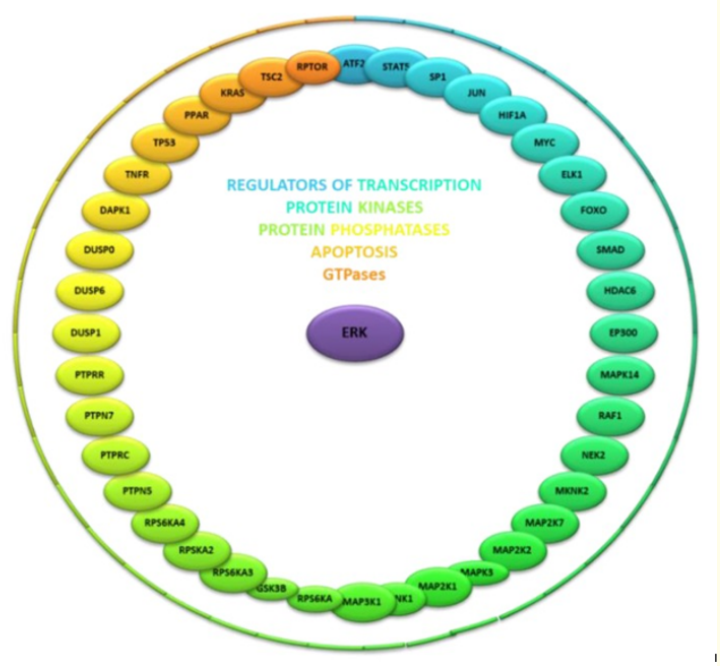

Figure 2: Courtesy ref no - Extracellular signal-regulated kinase (ERK) protein interactors and processes regulated by these interactors.

- Activation of transcription factor 2 (ATF2) by ERK2 Kinase facilitates growth, of intrahepatic cholangiocarcinoma, besides stimulation of apoptosis in the MDA-MB-435 breast cancer (BC) cells.
- Reduction In phosphorylation amounts of Signal Transducers and Activators of Transcription (STATs) in addition to ERK during imatinib therapy facilitates lymphopenia in chronic myelogenous leukemia (CML) cases,

- Inhibiting ERK1, E3LK1, HIH1A, vascular endothelial growth factor A (VEGFA) pathway represses, angiogenesis in cases of colorectal carcinoma [4].

- Lot of studies illustrated that activation along with stabilization of MYC transcription factor by ERK -based phosphorylation as oncogenic modes causing proliferation along with metabolic reprogramming in broad spectrum of cancer cells [4].

- $\quad$ Repression of Fork head box 03 (FOXO3) along with ERK phosphorylation at the time of glucosamine therapy reduces proliferation of A549 cancer cells [4]. Ursolic acid inhibits proliferation of colon cancer cells via inhibition of ERK phosphorylation,

- Besides amelioration of EP300- modulated acetylation of nuclear factor $\kappa \mathrm{B}(\mathrm{NF} \kappa \mathrm{B})$ in addition to CREB [4].

- Members of the RPS6K family were recently found to be the synthetic killer targets of combinational therapy with rapalog along with inhibitors of MAPK/ERK signaling pathways In triple negative breast cancer showing that ERK kinase is a Controller of protein translation [14].

- Hampering of the MAPK/ERK signaling avoided the ii) apoptosis stimulated via glycogen synthase kinase 3 B (GSK3B) inhibitors In case of acute promyelocytic leukemia treated with lithium [4].

- Hampering of the DUSP6 phosphatase utilizing BC1 inhibitors caused ERK kinase hyper activation, besides necrosis in malignant peripheral nerve sheath tumors [5].

- Conversely Protein Tyrosine Phosphatase Non-Receptor Type 7 (PTPN7) phosphatase facilitates propagation of glioma by the activation of ERK signaling [6].

- A germline mutation In DAPK1 death domain that disturbs stable crosstalk with ERK kinase In a yes association protein (YAP)-based way in addition to manipulate breast cancer cell apoptosis stimulated by ERK [7]. Crosstalk with Tuberous sclerosis and cancer (TSC2) in addition to RPTOR implies potential crosstalk with the mTOR signaling pathways. 
Escalation of Efficacy and Prevention of Chemoresistance in Various Cancer Therapies by the Utilization of Targeting the Crosstalk Amongst MAPK/ERK Along with Hippo/MST Signaling - A Comprehensive Review

Interactors Common to ERK as well as MST kinases-Identity as well as function

ERK kinase possesses lot of Interactors with the MST kinases that identifies the central kinase belonging to the Hippo signaling pathway (Figure 3). For controlling the action of these interactors it can be done by a positive along with negative manner. Calcyclinbinding protein (CACYPB), is part of a ubiquitinE3 complexes that takes part in the Ca-based ubiquitination in addition to proteasomal breakdown target substrates that have a part in controlling of proliferation of various cancer cells [8]. Crosstalk with CACYPB depicts probable mode of ERK as well as MST kinases control by ubiquitin break down. ii) Cul7 (cullin7) depicts other ERK as well as MST kinases interactors implicated in the event of protein ubiquitination. Cul7 represents a core part of $3 \mathrm{M}$ complex needed at the time of control of cytoskeleton dynamics in addition to Genomic integrity found to be the reason for hereditary human growth retardation syndrome. Control of Cul7 action occurs by mTOR signaling, that gives new proof with regards to the Control of ERK as well as MST kinases via mTOR signaling [9]. iii) BIRC5, that is an anti apoptotic protein was detailed as a target of Cul7, is implicated in the control of apoptosis. MST kinases that is understood to be controller of Genomic integrity at the time of apoptosis, crosstalk with Cul7, throws new light in this event in addition to implication of was not shown earlier. Transitional endoplasmic reticulum ATPase (VCP) facilitates sorting of protein at golgi apparatus level at a place where it works as a part of higher order controlling complexes. VCP takes part in the final step of ubiquitination along with ER correlated break down of 3 hydroxy -3 methyl-glutaryl -coenzyme reducatase (HMGCR) in a sterol based manner [10]. HMGCR is the rate limiting enzyme in cholesterol generation, that was earlier documented to be a controller of Hippo/MST signaling; Nevertheless, part of ERK kinase that in this event continues to be elusive. egl nine homolog 3 , from a family of proline hydroxylases (EGLN), depicts a significant cellular oxygen sensor taking part in the post translational control of Hypoxia inducible factor 1 (HIF 1), pointing to a probable association among cellular respiration, Reactive oxygen species (ROS) generation, besides controlling of ERK as well as MST kinases [11]. EGLN3 stimulates apoptosis. In cardiomyocytes in addition to neurons via interference with BAX -BCL2 complex followed by caspase 3 activation giving possible proof of ERK as well as MST kinases aiding each other at the time of control of apoptosis [12]. Hydroxylation of pyruvate kinase M (PKM) by
EGLN3activity halts glycolysis at the time of hypoxia causing crosstalk among EGLN3, ERK awa MST kinases as a mode of causing the control of metabolism [13]. Dephosphorylated form of eukaryotic translation initiation factor $4 \mathrm{E}$ binding protein 2 (EIF4EBP2) binds to EIF4E, besides avoiding EIF4F complex assembly, thus working as a repressor of translation [4]. Phosphorylation of EIF4EBP2 stimulates dislocation from EIF4E causing translation initiation [14]. It is properly understood that ERK as well as MST kinases are controllers of translation making EIF4EBP2, a target for both kinases. Furthermore EIF4EBP2 gets phosphorylated by with PI3K/ AKT/mTOR signaling pathway [4]. Myelin basic protein (MBP)is a part of the neuronal myelin membrane that converts events like remyelination of axons; it further controls T cell proliferation [4]. Crosstalk of ERK as well as MST kinases with MBP further gives insight in the part of these kinases in the control of neruron cells in addition to immune function. Nebulin binds along with stabilizes F-actin filaments, hence is thus significant for the sustenance of structural integrity of the cell [4]. In mechanotransduction, part of MST kinase had been earlier worked out but role of ERK kinase continues to evade us [4]. Crosstalk of ERK as well as MST kinases with exportin 6 (XPO6) depicts mode supporting nuclear - cytosol shuttlng of ERK as well as MST kinases in addition to highlighting the part of these kinases in RNA metabolism [4]. FOXO3, MYC along with TP53 proteins depict usual targets of ERK as well as MST kinases in case of cellular events like control of cellular metabolism, proliferation, in addition to apoptosis. Part of these events in ERK as well as MST signaling -later detailing. HDAC6 belongs to a family of Histone deacetylases taking part in deacetylation of lysine residues on the $\mathrm{N}$ terminal of core histones [4]. Furthermore HDAC6 participating in control of acetylation/deacetylation of proteins belonging to cytosol was also illustrated [4]. Control of the MST signaling by acetylation/deacetylation was previously detailed [16]. Part of ERK kinase in this event continues to be queried. Crosstalk of ERK as well as MST kinases with the action of caspases (caspase $3,-7,-8,-9)$ depicts one key mode of cell death controlled by these kinases-discussed later.

Interaction of MAPK/ERK pathway with rest of signaling pathways

ERK kinase controls the upstream part of MAP Kinase signal transduction pathway along with its by itself in feedback loops taking place in internal or external, positive or negative, controlling 


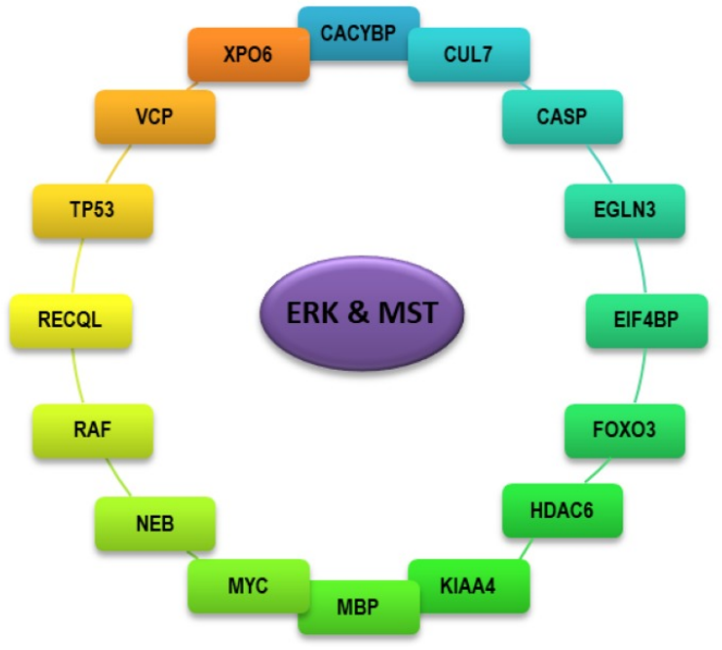

Figure 3: Courtesy ref no - Protein interactors shared between ERK and MST kinase.

feedback loops. In case of internal feedback loops, ERK ameliorates son of seven less (SOS) complex development along with In internal feedback loops, it stimulates ; the down stream interactors of growth factor receptor amounts. In external positive feedback loops, ERK ameliorates EGFR signaling activity, besides In external positive feedback loops it stimulates the growth factor facilitates signaling activity via external factors. This model partly gives reasoning of biostability in addition to oscillations In the ERK signaling as shown with the utilization of theoretical dynamic modeling conducted by Arkun., et al. [17] tuberous sclerosis tumor suppressor complex (TSC2) works at GTPase RHEB, that is a director of the mTORC1 Kinase that in turn stimulates S6K in addition to hampers 4EBP1 causing stimulation of protein translation along with protein metabolism [18]. Converse to this, hampering of TSC2 activity via phosphorylation of RPS6KA/RSK1 atS1798 was further illustrated [19]. RPS6KA/RSK1depicts an established Kinase that gets directly phosphorylated in addition to the activated by ERK Kinase but demonstrates the reverse action of TSC2 Control. A reduction in S6K phosphorylation at the activatory S424 following ERK in addition to RPS6KA/RSK1 depicts activation at the time of treatment with PI3K inhibitor GDC-0941was shown recently [19]. These out- comes emphasize on the probable interaction of ERK along with PI3KAKTwith different signaling pathways in the event of mTORC1 in addition to S6K Kinase activation. GSK3B depicts another kinase Controlled directly via ERK Kinase. This ERK Kinase directly binds along with phosphorylates GSK3B at T43 that causes activation of GSK3B, besides escalation of glucose metabolism that agrees with the action seen following phosphorylation of GSK3B by RPS6KA/ RSK1 [20]. These outcomes give a different proof of biphasic behavior in addition to oscillations in signaling. Details regarding proteins as well as aminoacid residues that get, phosphorylated via the MAPK/ERK signaling pathways is available at the Phospho site Plus database [21].

Mode regarding cell death stimulated by the MEK/ERK Kinase

Proliferation along with apoptosis in cancer cells gets blocked via hampering of MEK/ERK signaling. Conversely, various studies showed activation of MEK/ERK signaling is a crucial apoptotic mode stimulated by anti cancer drugs in cancer cells [22]. These findings are mostly based on the belief that a particular blocking of the MEK/ERK signaling interferes with the tumor growth hampering of in addition to cell death stimulation triggered by various drugs in cancer cell models. Induction of apoptosis stimulation via cisplatin in Hela in addition to HL-60 cells was based on the activation of the ERK signaling along with NOXA expression. Using MEK inhibitor U0126, besides ERK inhibitor peptide II to hamper inhibitors ameliorated NOXA expression, besides apoptosis secondary to cisplatin. Furthermore a reduction In amounts of anti apoptotic proteins MCL1 in addition to B cell lymphoma-XL (BCLXL) was detailed as ERK based [23]. The transient amounts of HRAS ${ }^{\mathrm{V12}}$ in HEK293T cells induced via NOXA expression along with BECLIN1autophagic cell death in an ERK based way [24]. Recovery of ERK signaling was necessary for heavy iron irradiation- stimulated multiple caspase getting activated at the time of glioma cell death. ERK signaling activation of along with that of caspase 3, -8, -9 induces apoptosis in T98G in addition to U251 glioma cell following heavy ion irradiation in addition to activation of caspases got reduced by the expression of the dominant negative form of ERK kinase in these cell lines [4]. Activation of caspase 3, -8, -9 In an ERK based manner was recently illustrated in T-ALL cells treated with PI3K inhibitor GDC0941 [41]. Even activation of caspase 9 by galectin 3 in addition to apoptosis induction by $\mathrm{T}$ cells was futher based on ERK signaling activation [25]. Further ERK along with NOTCH sig- 
Escalation of Efficacy and Prevention of Chemoresistance in Various Cancer Therapies by the Utilization of Targeting the Crosstalk Amongst MAPK/ERK Along with Hippo/MST Signaling - A Comprehensive Review

naling was detailed Controllers of activation of caspase 3, besides apoptosis induction in case of endothelial cells at the time chronic obstructive pulmonary disease (COPD) [4]. ERK kinase activation along with hampering of AKT Kinase results in stimulation of caspase 3 in various models of melanoma receiving therapy with 'acetoxy chavicol acetate analogue (ACA)28 modulator [26]. Moreover activation of ERK getting expressed by venom toxin bengaline also stimulated caspase 3, besides apoptosis induction in U937 leukaemic cells [4]. Reduction in the expression of BCL-XL along with MYC protein, besides activation of caspase $3,-8,-9$ in addition to apoptosis induced by natural alkaloid tryptanthrin, is based on the activation of ERK signaling in various leukemia cells [52]. Interaction among caspase -8, Reactive oxygen species (ROS) along with ERK signaling was evaluated as a mode manipulating the phagocytosing of neutrophils fate [27]. ERK signaling stimulation along with caspase 8 activation occurred secondary toinducible form of RAF in HEK293 cells [4]. Moreover a key part of caspase 8 in EGF signaling was further illustrated [28]. Even the cytotoxicity of thujaplicin in Hepatocellular carcinoma (HCC) was ascribed to the ERK signaling activation [4]. Hampering ERK signaling ameliorated changes In the BCL2 family of proteins getting expressed, in addition to hyper activation of caspase $3,-7$, along with apoptosis in lung cancer getting therapy with quercetin [4]. Causing hyper activation of ERK signaling by multiple modes causes toxicity to the RTS-RAF mutations- stimulated lung adenocarcinoma cells [4]. Autoactivating ERK 2 kinase mutants expression In prostate cancer cells results In stimulation of cell cycle arrest without any dependence on RAF signaling [4]. Apoptosis induction, besides autophagy in osteosarcoma cells by biphenolic compound honokiol is further based on activation of ERK signaling [29]. ERK signaling also possesses key part at the time of activation of caspase $3,-8$, in addition to autophagic cell death that got stimulated by 2 amininicitinonitrile (wo9) in gastric cells [30]. Maintained activation of ERK was necessary for the hampering of proliferation along with G0/G1 -phase cell cycle arrest in human, carcinoma cells having therapy with farreol [4]. Further ERK manifests cell cycle arrest in addition to senescence in prostate neoplasm, besides blocking the conversion of primary oncogenic RAS [28]. Stimulation of ERK signaling represses growth of effector $\mathrm{T}$ cells that have been antigen-hyper stimulates. Biphasic activation of ERK-MSK1 signaling Controls apoptosis stimulated by DNA injury [4]. These outcomes make ERK kinase a probable tumor repressor
Mode of cell death mediated via the Hippo/MST signaling pathway

The Hippo signaling regulates the formation in addition to organ size in separate type of species. Impairment of this pathway stimulates tumor generation In case of model organisms along with taking place in a lot variety of human carcinomas [4]. MST1/2 serine/threonine kinases are the central controllers of the Hippo signaling pathway. Hampering of cellular proliferation takes place by the stimulation of MST1/2 kinases, that promotes apoptosis in various cancer cells addition to model organisms, thus working as a tumor repressor [4]. A lot of modes are used in common by Hippo/MST along with MAPK/ERK signaling pathway with regards to the control of proliferation of cells in addition to stimulation of apoptosis. DNA binding proteins TEA domain (TEAD) activity along with expression of MYC oncogenesin T-ALL in addition to breast cancer cells are reduced via MST signaling in xenograft mice model of breast cancer [31]. Glucose metabolism is controlled through MST kinases In T-ALL, laryngeal squamous in addition to breast cancer cells via hampering TEAD transcriptional activity along with expression of glucose transporter (GLUT) [31]. Stimulation of apoptosis occurs by MST kinases via utilization of expression of proapoptotic protein NOXA in a variety of cancer cells via phosphorylation in addition to the activation of Fork head box 0 (FOXO) transcription factors [32]. Direct phosphorylation in addition to activation of proapoptotic protein BIM, caspase 3, -9 along with apoptosis in case of pancreatic $\beta$ cells [33]. Furthermore Hippo/MST signaling hampers the expression in addition to activation of various anti apoptotic protein like IAP, MCL along with BCL-XL. MST further activates caspases in addition to caspases potentiate its proapoptotic activity [34]. Furthermore caspase-8 activation by the Hippo/MST signaling was further illustrated [19]. Crosstalk among caspase-8 in addition to ERK signaling depicts a crucial mode in the EGFR signaling. Ultimately, a computational model that anticipated diverse dynamic profiles regarding the Hippo- ERK crosstalk network get developed [35].

\section{Hippo/MST signaling pathway activation in cancer cells}

Both Hippo/MST signaling along with ERK signaling pathway possess various common targets for controlling proliferation in addition to cell death In cancer cells (Figure 4). Hippo/MST signaling activation was show to be the key mode implicated In action of different anticancer agents. From all these outcomes it is 
pointed that there is synergistic action among inhibitors in addition to activators of ERK signaling along with activators of Hippo/ MST signaling for cancer treatment. Phosphorylation of MST by AKT kinase at T120 in addition to inhibition of MST action occurs [36]. Once there is targeted inhibition of the PI3K/AKT/mTOR signaling axis it stimulates activation of the MST kinases, besides inhibition of YAP effector in a wide kind of cancer cells. T-ALL cells getting treatment with the utilization of PI3K inhibitors GDC-0941 resulted in MST 1kinase, ERK kinase in addition to apoptosis [47]. Repression of cell growth, besides inhibition by LY94002, In case of castration resistant C4-2prostate cancer along with HCT 116 colon cancer cells [36]. YAP activation in addition to MYC expression gets blocked by Wortmannin, that is modulated by EGF in HCC, besides mammary epithelial cells [36]. Combination PI3K/mTOR inhibitors with Fibroblast growth factor receptor4 (FGFR4) inhibitors BLU9931 potentiates MST1/2 activation in addition to stimulates apoptosis in HER2 breast cancer. Pan mTOR inhibitors MLN0128 activates caspase3, -7 along with facilitates apoptosis in intrahepatic cholangiocarcinoma stimulated In mice via over expression of YAP [37]. Tesrolimus, that is a rapamycin obtained agent stimulates YAP protein breakdown through of autophagy in human angiomyolipoma [38]. Various natural compounds possessing anticancer action got detailed as robust activators of MST kinase in cancer cells. Shikonin that is a naphthoquinonic agent is implicated In YAP -TEAD crosstalk via the activation ERK as well as MST signaling In T-ALL cells [29]. Fisetin, a flavomnol stimulates LATS along with ERK kinases, besides apoptosis induction In human osteosarcoma cells [39]. Curcumin, a polyphenolic agent stimulates cell cycle arrest, autophagy in addition to generation of Reactive oxygen species, activation of MST kinase, ERK kinase, caspase3, -9 along with down regulation of YAP protein in different cancer cell models [40]. Once the Hippo/YAP signaling, that is oncogenic gets activated via the activation of tumor Suppressor LKB1 through honokiol, it ameliorates breast tumor growth in addition to metastasis In cancer cells, Various other drugs along with agents were detailed as the activators of the Hippo/MST signaling In cancer cells in mice [41]. Activation of MST as well as ERK signaling In T-ALL cells occurs via Vitamin E analogues besides breast cancer cells that resulted in apoptosis induction [4]. HMGCR inhibitors, a rate limiting step in mevalonate biosynthesis represses malignant mesotheloma cells via blockade of YAP/CD44 growth stimulatory axis [42]. Large tumor-suppressor kinase (LATS) kinase phosphorylation as well as YAP protein break down gets stimulated through pyaocoumarin (Decursin) via activation of TRCP ubiquitin E 3 ligase In case of Hepatocellular carcinoma (HCC) [43,44] (Figure 5). Atriterpine which is a tetracyclic, namely Cucurbitacin B stimulates apoptosis by activation of LATS kinase besides caspase 8 activation besides caspase 8 activity In colorectal cancer cells [45]. YAP/TEAD crosstalk gets interfered by flavones Apigenin in addition to causing reduction in viability, besides migration of triple negative breast cancer cells along with tumor development in vivo [46].

Targeting MAPK/ERK, PI3K/AKT/mTOR as well as Hippo/MST pathway in combination in cancers

In case of cancer cell models, trying targeted inhibition of MAPK/ERK, signaling pathway stimulates growth repression in addition to cell lysis. Ultimate action of the MAPK/ERK control is on the basis of the inter action occurring with rest of the signaling pathways that are active in case of cancer cells. RAS/RAF signaling stimulates PI3K/AKT along with MEK/ERK signaling pathway, besides might get inhibited by ERK along with AKT kinase in the feedback loop. Variety of targeted inhibitors of RAF kinase have been Clinically evaluated for different kinds of cancers, that are

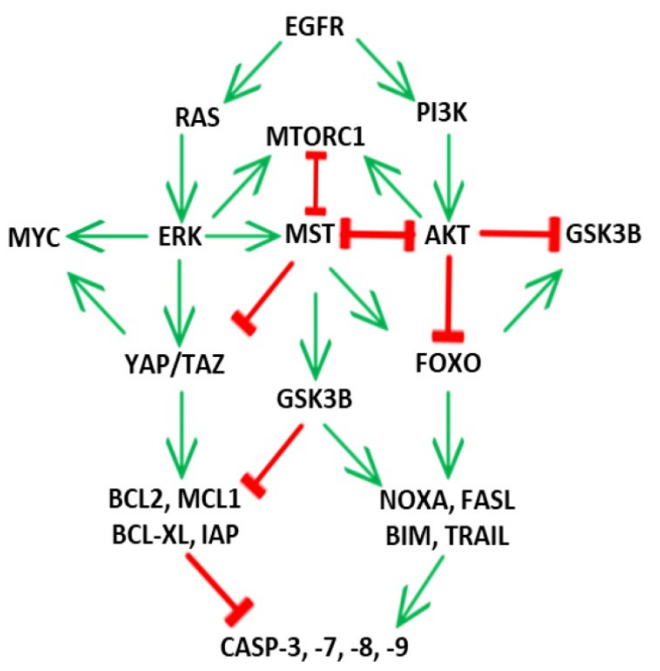

Figure 4: Courtesy ref no - Cross-talk of the mitogen-activated protein kinase (MAPK)/ERK signaling pathway and mechanism of cell death induction through the ERK-Hippo interplay. 


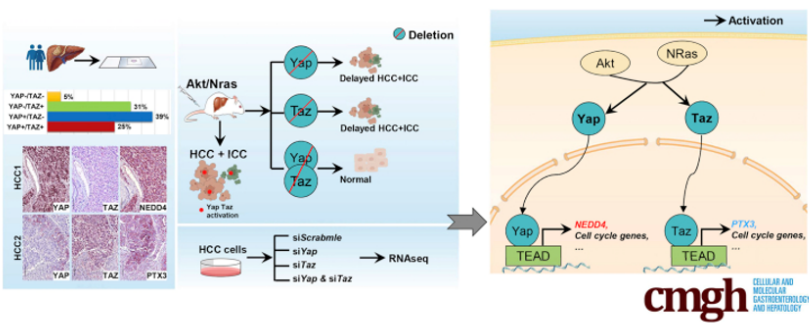

Figure 5: Courtesy ref no - 108-Yes-associated protein (YAP) and transcriptional co-activator with post synaptic density protein, drosophila disc large tumor suppressor and zonula occludens-1-binding motif (TAZ) coordinately regulate liver cancer development. Human liver tumors show ubiquitous activation of YAP or TAZ, thus relying on either YAP or TAZ for their growth.

inclusive of balvarafenib, dabrafenib, in addition to LY3009120 (1-(3,3-dimethylbutyl)-3-(2-fluoro-4-methyl-5-(7methylamino) pyrido[2-3d]pyrimidin-6yl)phenyl)urea) [47]. Nevertheless, stimulation of MEK/ERK kinase by modes separate from RAF signaling in addition to generation of cancer resistance at the time of mono therapy with a single RAF kinaseinhibitor usually takes place. Thus combination of RAF targeted inhibitors along with those of MEKkinase (trametinib, binimetinib along with U0126), in addition to ERK kinase (ulixertinib, SCH77964, along with LY3214996) might markedly enhance the result of cancer therapies (Figure 6) [47]. At the time of targeted blockade of PI3K/AKT along with MAPK/ ERK signaling pathways in different cancer cells, stimulation of the Hippo/MST signaling pathways acts as a significant mode of cell death induction [4]. Various synthetic (C19), along with natural agents were documented to be robust stimulators of Hippo/MST signaling in a wide range of cancer cells. Targeting Hippo/MST signaling stimulators in addition to PI3K/AKT as well as MEK/ ERK In combination might behaving the capacity of getting over the resistance in cancer cells, besides escalation of effectiveness of treatment as demonstrated recently [48]. High risk neuroblastomas getting away from the particular pressure of MEK inhibition might possess sensitivity to combination of treatments that target Hippo/MST as well as MEK signaling [49]. Targeting MAPK/ERK in addition to Hippo/MST signaling PI3K pathway has proven to be a new treatment method for uveal melanoma. Stimulation of the Hippo/MST signaling might prove to be of advantage at the time of inhibition of pancreatic ductal adenocarcinoma generation that got treated with U0126, besides LY294002 inhibitors. The Stimulation of the Hippo/MST signaling hampers YAP based transcription that causes a reduction in expression of MYC, BCL2, BCLXL, as well as MCL1anti apoptotic proteins, besides induction of caspase-based cell death. Combining therapy with BH3 mimetics, the particular inhibitors of BCL2, BCLXL, as well as MCL1anti apoptotic proteins with (obatoclax, navitoclax as well as venetoclax) in addition to particular inhibitors of MYC transcription action (10058-F4 as well as 10074-G5) comprises a different advantageous approach for enhancement of targeted treatment dependent on PI3K/AKT/mTOR in addition to MAPK/ERK inhibitors (Figure 6) [4]. Hampering of MAPK/ERK signaling with the utilization ofPD98059that gets over the Resistance to BH3 mimetics obatoclax in small cell lung cancer [48]. BH3 mimetics resulted in reduction of viability of melanoma cells that demonstrated relapse following therapy with BRAF/MEK inhibitors [50]. BH3 mimetics attain synergy with the MEK kinases inhibitor U0126, at the time of apoptosis induction in melanoma cells [51]. BH3 mimetic polyphenol(-)-Gossypol(AT-101) resulted in activation of ERK signaling along with apoptosis in malignant mesothelioma (MM) cells, besides In mice delivered intraperitoneally transplanted with mouse MM cell lines Stimulation of the MEK/ERK signaling in addition to PI3K signaling hampering got documented for stimulation of cell death In different cancer cells [52]. MEK/ERK signaling pathways work as probable tumor Suppressing, with controlling proliferation in addition to cell death in case of cancer cells [4]. Hyperactivation of the ERK signaling hampers proliferation, besides causing stimulation of apoptosis In different types of B-Raf (V600E) cancer cells [53]. In these kind of subjects, targeted hampering of MEK/ERK signaling represses cell death, besides resulting in the generation of chemoresistance in case of cancer cells [54]. Thus, a proper combination MAPK/ERK, in addition to PI3K/AKT/mTOR inhibitors along with stimulators of Hippo/MST signaling, BH3 mimetic as well asinhibitors of MYC transcription action can result in escalation of present targeted treatments.

\section{Conclusions}

The presence of double kind of action in MAPK/ERK signaling possesses a remarkable effect on the Clinical results of the MAPK/ 


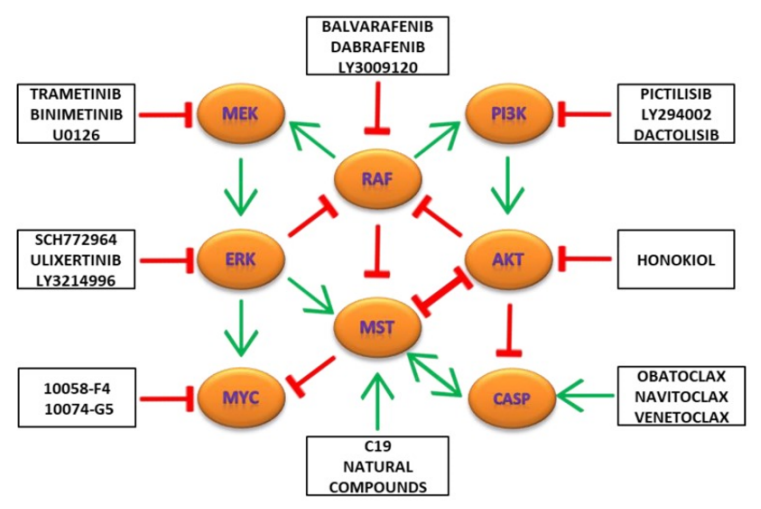

Figure 6: Courtesy ref no - 7 - Targeting of the MAPK/ERK-Hippo/MST-PI3K/AKT network for effective cancer therapy.

ERK targeted treatments. Combining PI3K/AKT/mTOR in addition to MEK/ERK inhibitors can probably cause a reduction in the effectiveness secondary to separate influences on the Hippo/MST signaling pathway. Whereas active PI3K/AKT/mTOR signaling possesses a negative influences on the Hippo/MST action, stimulation of signaling further causes the escalation of the cancer cell death via the Hippo/MST signaling pathway. Dependent on these findings, hampering of the combination of MAPK/ERK pathway at RAF in addition to MEK/ERK kinase levels appears to possess greater effectiveness in cancer cells based purely on the action of MAPK/ ERK signaling pathway. Attempting reactivation of the MEK/ERK axis In cancer cells that have fallen in addiction with hampering of the Hippo/MST signaling pathway via the PI3K/AKT/mTOR signaling pathway might prove to be of advantage following targeted blocking. Furthermore stimulation of the Hippo/MST signaling as well as chemical controlling of the downstream targets can impose significant escalation of the Clinical results of the targeted treatments that are dependent on MAPK in addition to PI3K signaling inhibitors. Properties of the MEK/ERK Controlling axis further guides towards the therapies of various other pathological conditions like Metabolic Syndrome (MetS), other occasional genetic diseases like neurodegenerative diseases, (NGD) like myotonc dystrophy type1 [55].
Bibliography

1. Burotto M., et al. "The MAPK pathway across different malignancies: a new perspective". Cancer 120.22 (2014): 34463456.

2. Chappell WH., et al. "Ras/Raf/MEK/ERKand with PI3K/ PTEN/AKT/ mTOR:rationale and imortanc of inhibiting these pathway in human health". Oncotarget 2 (2011): 135-164.

3. Oughtred R., et al. "The. BioGRID interaction databases: 2019 update". Nucleic Acids Research 47 (2019): D529-D541.

4. Valis K and Novak P. "Targeting ERK- Hippo interplay in cancer therapy". International Journal of Molecular Sciences 21 (2020): 3236.

5. Ramkisson A., et al. "Targeted inhibition of the dual specificity phosphatases DUSP1and DUSP6 suppress MPNST growth via JNK". Clinical Cancer Research 25 (2019): 4117-4127.

6. Jin T., et al. "PTPN7 promotes the progression of glioma by activating the MAPK/ERK and the PI3K/ AKT pathways and is associated with poor patient survival". Oncology Report 42 (2019): 717-723.

7. Stevens C., et al. "A germline mutation In the death domain of DAPK1 inactivates ERK induced apoptosis". Journal of Biological Chemistry 282 (2007): 13791-13803.

8. Lian YF, et al. "CACYPB enhances cytoplasmic reduction of P-27 (Kip1) to promote Hepatocellular carcinomaprogression in the absenc of RNF41 mediated degradation". Theranostics 9 (2019): 8392-408.

9. YuQ., et al. "Gosspol inhibits cullin neddylation by targeting SAG-CUL5 and RBX-CUL1 complexes". Neoplasia 22 (2020): $179-191$

10. LeichnerGS., et al. "HMG-CoA reducatase and Insig 1, two polytopic endoplasmic reticulum proteins, en route to proteasomal degradation". Molecular Biology of the Cell 20 (2009): 33303341.

11. Gastelum G., et al. "Restoration of the prolyl - hydroxylase domain protein-3 oxygen sensing mechanism is responsible for regulation of HIF 2 alpha expression and induction of sensi- 
tivity of myeloma cells to Hypoxia -mediated apoptosis". PLoS ONE 12 (2017): e188438.

12. Hogel H., et al. "Prolyl hydroxylase PHD3 enhances the Hypoxic survival and G1 to S transition of, carcinoma cells". PLoS ONE 6 (2011): e27112.

13. Schoepflin ZR., et al. "PHD3 is a transcriptional coactivator of HIF 1 alpha in nucleus pulposus cells independent of the PKM2-JMJD5 axis". FASEB Journal 31 (2017): 3831-3847.

14. Hulea L., et al. "Translational and HIF 1 alpha dependent metabolic reprogramming underpin metabolic plasticity and responses to kinase inhibitors and biguanides". Cell Metabolism 28 (2018): 817-832e8.

15. Ding M., et al. "The mTOR targets $4 \mathrm{E}-\mathrm{BP} 1 / 2$ Restrain tumor growth and promote Hypoxia tolerance in PTEN driven prostate cancer". Molecular Cancer Research 16 (2018): 682-695.

16. ZanconatoF., et al. "YAP/TAZ at the roots of cancer". Cancer Cell 29 (2016): 783-803.

17. Arkun Y and Yasemi D. "Dynamics and control of ERK signaling pathway: sensitivity, biostability and oscillations”. PLOS ONE 13 (2018): e0195513.

18. Wang J., et al. "A non canonical MEK/ERK signaling pathway regulates autophagy via regulating Beclin 1". Journal of Biological Chemistry 183 (2009): 7388-7397.

19. Roux PP., et al. "Tumor promotingphorbol esters and activated Ras inactivates the tuberous sclerosis tumor suppressor complex via p90 ribosomal S6 Kinase". Proceedings of the National Academy of Sciences of the United States of America 101 (2004): 13489-13494.

20. Ding Q., et al. "Erk associates with and primes GSK3beta for its inactivation resulting in up regulation of beta-catenin". Molecular Cell 19 (2005): 159-170.

21. Hornbeck PV., et al. "Phospho site: A bioinformatics resource dedicated to physiological proteins phosphorylation". Proteomics 4 (2004): 1551-1561.

22. Tsuiko 0., et al. "A speculative outlook on embryonic aneuploidy: can molecular pathways be involved?" Developmental Biology 447 (2019): 3-13.
23. Sheridan C., et al. "An ERK - dependent pathway to Noxa expression regulates apoptosis by platimum -based chemotherapeutic drugs". Oncogene 29 (2010): 6428-6441.

24. Elgendy M., et al. "Oncogenic Ras -induced expression of Noxa and Beclin -1promotes autophagic cell death and limits clonogenic survival". Molecular Cell 42 (2011): 23-35.

25. Xue H., et al. "The N-terminal tail coordinates with carbohydrate recognition domain to mediate galectin 3 induced apoptosis in Tcells". Oncotarget 8 (2017): 49824-9838.

26. Satoh R., et al. "Identification of ACA-28, a1'acetoxychavicol acetate analogue compound, as a novel modulator of ERK MAPK signaling which preferentially kills melanoma cells". Gene Cells 8 (2017): 22-608-618.

27. Pathania AS., et al. "The synthetic tryptanthrin analogue suppresses STAT3 signaling andinduces caspase dependent apoptosis via ERK upregulation In human leukaemia HL-60cells". PLoS ONE 9 (2014): e110411.

28. Zhang G., et al. "Beta thujaplicin induces autophagic cell death, apoptosis and cell cyclearrest through ROS mediated Akt and p38/ERK MAPK signaling in Hepatocellular carcinoma”. Cell Death and Disease 10 (2019): 255.

29. Huang K., et al. "Honokiol induced apoptosis and induced autophagy via the ROS/ERK1/2 signaling pathway in human osteosarcoma cells in vitro and in vivo". Cell Death and Disease 9 (2018): 157.

30. Zhang P., et al. "w09, a novel autophagy enhancer, induces autophagy dependent cell apoptosis via activation of the EGFR mediated RAS-RAF1-MAP2K-MAPK1/3pathway". Autophagy 13 (2017): 1093-1112.

31. Valis K., et al. "Shikonin regulates C-MYC and GLUT1 expression through the MST1-YAP-1-TEADaxis". Experimental Cell Research 349 (2016): 273-281.

32. Valis K., et al. "Hippo/Mst1 stimulates transcription of the pro- apoptotic mediator NOXA in a Fox01- dependent manner". Cancer Research 71 (2011): 946-954.

33. Ardestani A., et al. "MST1 is a key regulator of beta cell apoptosis and dysfunction in diabetes". Nature Medicine 20 (2014): 385-397.

Citation: Kulvinder Kochar Kaur., et al. "Escalation of Efficacy and Prevention of Chemoresistance in Various Cancer Therapies by the Utilization of Targeting the Crosstalk Amongst MAPK/ERK Along with Hippo/MST Signaling - A Comprehensive Review". Acta Scientific Cancer Biology 5.9 (2021): $37-47$. 
34. Smoot RL., et al. "Platelet derived growth factor receptor regulates YAP-1 transcriptional activity via Src family kinase dependent tyrosine phosphorylation". Journal of Cellular Biochemistry 119 (2018): 824-836.

35. Collak EK., et al. "Threonine 120 phosphorylation regulated by phosphatidyl inositide 3-kinase/Akt and mammalian target of rapamycin pathway signaling limits the anti tumor activity of mammalian Sterile 20-like kinase 1". Journal of Biological Chemistry 287 (2012): 23698-23709.

36. Xia H., et al. "EGFR-PI3K, -PDK1 pathway regulates YAP signaling In Hepatocellular carcinoma:the mechanisms and its implications in targeted therapy". Cell Death and Disease 9 (2018): 269.

37. Zhang S., et al. "Pan mTOR inhibitorsMLN0128 is effective in intrahepatic cholangiocarcinoma In mice". Journal of Hepatology 67 (2019): 1194-1203.

38. Liang N., et al. "Regulation of YAP by mTOR and autophagy reveals a therapeutic tuberous sclerosis complex". Journal of Experimental Medicine 211 (2014): 2249-2263.

39. Valis K., et al. "Reprogramming of leukemic cells metabolism through the naphthoquinonic compound Quambalarine B". Oncotarget 8 (2017): 193137-193153.

40. Gersey ZC., et al. "Curcumin decreases malignant characteristics of glioblastoma stem cella via induction of Reactive oxygen species". BMC Cancer 17 (2017): 99.

41. Sengupta S., et al. "Activation of tumor Suppressor LKB1 by honokiol abrogates cancer stem -like phenotype in breast cancer via inhibition of Oncogenic Stat3". Oncogene 36 (2017): 5709-5721.

42. Tanaka K., et al. "Statin Suppresses Hippo pathway-inactivated malignant mesotheloma cells and blocks the YAP /CD44 growth stimulatory axis". Cancer Letter 385 (2017): 215-224.

43. Li J., et al. "Decursin inhibits the growth of HepG2 Hepatocellular carcinoma cells via Hippo/ YAP signaling pathway". Phytotherapy Research 32 (2018): 2456-2465.

44. Wang H., et al. "Distinct and Overlapping Roles of Hippo Effectors YAP and TAZ During Human and Mouse".
45. Chai Y., et al. "Cucurbitacin B inhibits the Hippo-YAP signaling pathway and exerts anticancer activity In colorectal cancer cells". Medical Science Monitor 24 (2018): 92451-92458.

46. Li YW., et al. "Apigenin Suppresses the stem cell-like properties of triple negative breast cancer cell by inhibiting YAP/TAZ activity". Cell Death Discovery 4 (2018): 105.

47. Yaeger R and Corcoran RB. "Targeting alterations In the RAFMEK pathway". Cancer Discovery 9 (2019): 329-341.

48. Feng R., et al. "MAPK and Hippo signaling pathways crosstalk via the RAF1/MST 2interaction in malignant melanoma". Oncology Report 38 (2017): 1199-1205.

49. Coggins GE., et al. "YAP1 mediates resistance to MEK1/2 inhibition in neuroblastomas with hyperactivated RAS signaling". Cancer Research 79 (2019): 6204-6214.

50. Li H., et al. "YAP-TAZ activation drives uveal melanoma initiation and progression". Cell Report 29 (2019): 3200-3211. e4

51. Mukherjee N., et al. "BH3 mimetics induce apoptosis independent of DRP1 in melanoma". Cell Death and Disease 9 (2018): 907.

52. Verhagen M., et al. "A novel BH3 mimetic reveals a mitogen activated protein kinase dependent mechanisms of melanoma cell death Controlled by p53 and Reactive oxygen species". Cancer Research 66 (2006): 11348-11359.

53. Atiq R., et al. "Suppression of B-Raf (V600E) cancers by MAPK hyper activation". Oncotarget 7 (2016): 18694-18704.

54. YuS., et al. "ERK 1 indicates a good prognosis and inhibits breast cancer progression by m Suppressing YAP1 signaling". Aging 11 (2019): 12295-122314.

55. Kulvinder Kochar Kaur., et al. "Probable Crucial Role of Alteration In Insulin Signaling inthe Pathophysiology of Myotonicdystrophy type1-A Systemic Review". Journal of Endocrinology and Metabolism 1.2 (2020): 1-21.

\section{Volume 5 Issue 9 September 2021}

(C) All rights are reserved by Kulvinder Kochar Kaur., et al. 